\title{
Las competencias del talento en la Industria 4.0, demanda vs oferta: caso de estudio de la Universidad Rey Juan Carlos, España
}

\author{
María-Carmen De-la-Calle-Durán, José-Luis Rodríguez-Sánchez y Thais González-Torres \\ Dpto. Economía de la Empresa (ADO), Economía Aplicada II y Fundamentos del Análisis Económico, Universidad Rey \\ Juan Carlos, Paseo de los Artilleros s/n, Madrid - España. (Correo-e: carmen.delacalle@urjc.es; \\ joseluis.rodriguez.sanchez@urjc.es; thais.gonzalez@urjc.es)
}

Recibido Jun. 9, 2021; Aceptado Jul. 29, 2021; Versión final Sep. 21, 2021, Publicado Feb. 2022

\section{Resumen}

Este estudio tiene como objetivo identificar el grado de concordancia entre las competencias que se demandan en la Industria 4.0 y las competencias que la Universidad Rey Juan Carlos (Madrid, España) establece en los itinerarios formativos del plan de estudio del grado de administración y dirección de empresas. La evolución de los puestos de trabajo y las competencias para la Industria 4.0 ha despertado el interés tanto de investigadores como de directivos de las empresas. Para desarrollar este estudio, se ha realizado una revisión de la literatura científica, además de la consulta de documentos internos de la propia universidad. Los resultados revelan divergencias entre las necesidades de la industria en términos de competencias y las adquiridas por los alumnos en su formación universitaria. Se concluye que se debe hacer una profunda reflexión para incorporar ciertas competencias técnicas, metodológicas y personales que son clave en el entorno actual de la Industria 4.0.

Palabras clave: industria 4.0; competencias; habilidades; talento; educación universitaria

\section{Industry 4.0 talent skills, demand versus supply: case study at the Rey Juan Carlos University, Spain}

\begin{abstract}
The main objective of this study is to identify the degree of concordance between competences demanded by Industry 4.0 and the competences that the Universidad Rey Juan Carlos (Madrid, Spain) includes in its training itineraries for the degree of business administration and management. The evolution of Industry 4.0 jobs and skills has increased the interest of both researchers and firm managers. A review of the scientific literature is performed along with a review of the university's internal documents. The results reveal divergences between the skills demanded by the Industry 4.0 and the skills acquired by students during their university careers. It is concluded that study programs require profound changes to incorporate specific technical, methodological, and personal competences that are essential in the current Industry 4.0 environment.
\end{abstract}




\section{INTRODUCCIÓN}

La cuarta Revolución Industrial (4RI), también conocida como Industria 4.0, hace referencia a un nuevo paradigma basado en la tecnología, en donde los sistemas de información y comunicación se utilizan, en gran medida, para mejorar la productividad. Convergen las tecnologías digitales, físicas y biológicas, por medio de la inteligencia artificial, el internet de las cosas, el Big Data, la computación en la nube, la biotecnología y los nuevos modelos de negocio (Schuh et al., 2014). La Industria 4.0 describe la creciente digitalización de toda la cadena de valor y la consiguiente interconexión de personas, objetos y sistemas mediante el intercambio de datos en tiempo real (Hecklau et al., 2016).

La introducción de las últimas tecnologías digitales y de automatización en la fabricación, así como los nuevos valores sociales de los empleados y los cambios demográficos, van a afectar significativamente a los procesos de trabajo y al entorno laboral (Chryssolouris et al., 2013). Conlleva la transición a una economía que se fundamenta en el conocimiento influyendo directamente en dimensiones como la educación y la formación de las nuevas generaciones. Por ello, desde el ámbito de la educación universitaria se requiere prestar atención a las competencias necesarias en la Industria 4.0 ya que, es en esta etapa, donde las personas se capacitan para ingresar a la vida laboral. Las instituciones de educación superior necesitan enfocar sus esfuerzos en brindar todas las habilidades necesarias a los estudiantes, no solo para el presente sino también para enfrentar el trabajo del futuro.

En este nuevo entorno, las tareas rutinarias, repetitivas y automatizadas serán ejecutadas por máquinas o robots, mientras que las tareas que requieren experiencia, intuición, creatividad o toma de decisiones basadas en la incertidumbre seguirán residiendo en las personas y su talento, lo cual se considera clave en esta cuarta revolución industrial (Fitsilis et al., 2018). Así, el papel de los trabajadores evolucionará hacia la gestión de las actividades realizadas por los robots y las máquinas inteligentes que cooperan con ellos. Esto reducirá la demanda de trabajadores con baja cualificación a la vez que se incrementará la demanda de empleados que realicen actividades de supervisión (Saniuk et al., 2021). Los cambios a los que hay que hacer frente exigen talento, habilidades y competencias que son muy diferentes a las necesidades de las tres revoluciones industriales anteriores (Grzelczak et al., 2017; Maisiri et al., 2019). Estas nuevas exigencias han provocado una brecha entre lo que las empresas necesitan y los perfiles profesionales que están disponibles en el mercado laboral, lo que supone pérdidas económicas y gastos de formación adicionales (Goulart et al., 2021).

La evolución de los puestos de trabajo y las competencias para una implementación exitosa de la Industria 4.0 ha despertado recientemente el interés tanto de investigadores como de los directivos de las empresas, así como de los responsables en materia de educación en el ámbito universitario. Sin embargo, la investigación en este ámbito sigue siendo escasa (Pinzone et al., 2017; Moldovan, 2019). Estudiar los requisitos o exigencias de habilidades o competencias en la era de la Industria 4.0 es de importancia no solo para quienes buscan empleo, sino también para las empresas y las instituciones educativas (Maisiri et al., 2019).

Analizada la literatura existente en el tema de investigación, se observa como trabajos recientes como el de Saniuk et al. (2021) estudian desde un enfoque generalista las áreas clave de conocimiento y habilidades requeridas para implementar el concepto de Industria 4.0. Goulart et al. (2021), por su parte, evalúan la relación entre el perfil profesional requerido por las empresas de tecnologías de la información (TI) y lo que se enseña a los estudiantes en los programas relacionados con las TI en las instituciones de educación superior. De manera más concreta, Rivera et al. (2021) identifican las habilidades de innovación que los perfiles de ingeniería deben adquirir en la actual industria. Sin embargo, no existen trabajos centrados en perfiles no directamente relacionados las $\mathrm{TI}$.

Con el objetivo de completar esta laguna, se lleva a cabo un análisis comparativo entre las competencias que se demandan en la Industria 4.0 y las competencias que la Universidad Rey Juan Carlos (URJC) establece en los itinerarios formativos del plan de estudio del grado de Administración y Dirección de Empresas (ADE) y sus dobles grados asociados. De manera más concreta, se pretende analizar el grado de concordancia entre las competencias demandadas por la Industria 4.0 y las que se desarrollan en la universidad. Estos objetivos se recogen en las siguientes preguntas de investigación: i) ¿Cuáles son las principales competencias y habilidades que exige la Industria 4.0.?; ii) ¿Cuáles son las principales competencias que los alumnos de Administración y Dirección de Empresas y sus dobles grados asociados deben desarrollar durante sus estudios?; iii) ¿Existen diferencias entre las necesidades de la industria en términos de competencias y las adquiridas por los alumnos en su formación universitaria?

\section{MATERIALES Y MÉTODOS}

Para desarrollar este estudio se ha realizado una revisión de la literatura científica. Las bases bibliográficas empleadas han sido Web of Science (WoS) y Scopus. Ambas están especializadas en el ámbito de las Ciencias Sociales. Además, nos permiten filtrar la búsqueda por categorías y ver el número de veces que dicho documento aparece citado en la base de datos entre otras funciones, lo que nos permite analizar su relevancia. El protocolo de revisión de la literatura científica es mostrado en la Tabla 1. 
Tabla 1. Protocolo de revisión de literatura científica

\begin{tabular}{|c|c|c|}
\hline Base de Datos & WoS & Scopus \\
\hline Alcance geográfico & Bibliografía científica mundial & Bibliografía científica mundial \\
\hline Características & $\begin{array}{l}\text { Indicadores de calidad: } \\
\text { Factor de impacto JCR } \\
\text { Índice de inmediatez } \\
\text { Número de citas } \\
\text { Cuartiles }\end{array}$ & $\begin{array}{l}\text { Indicadores de calidad: } \\
\text { Factor de impacto SJR } \\
\text { Número de citas } \\
\text { Cuartiles }\end{array}$ \\
\hline Criterios de búsqueda & Tema & Título del artículo, resumen y palabras clave \\
\hline Rango de datos & Todos los años hasta 2021 & Todos los años hasta 2021 \\
\hline Fecha de búsqueda & 14 septiembre 2021 & 14 septiembre 2021 \\
\hline Términos de búsqueda & $\begin{array}{l}\text { "Industry 4.0" AND } \\
\text { "Competences" AND “Skills" }\end{array}$ & $\begin{array}{l}\text { "Industry 4.0" AND } \\
\text { "Competences" AND "Skills" }\end{array}$ \\
\hline Criterios de inclusión & $\begin{array}{l}\text { Artículos de revista } \\
\text { Capítulos de libro }\end{array}$ & $\begin{array}{l}\text { Artículos de revista } \\
\text { Capítulos de libro }\end{array}$ \\
\hline Número de documentos & 44 & 57 \\
\hline $\begin{array}{l}\text { Proceso de filtrado: } \\
\text { Categorías }\end{array}$ & $\begin{array}{l}\text { "Education Educational Research" } \\
\text { "Management" }\end{array}$ & $\begin{array}{l}\text { "Business, Management and Accounting" } \\
\text { "Economics, Econometrics and Finance" }\end{array}$ \\
\hline Número de documentos & 18 & 22 \\
\hline Proceso de filtrado & $\begin{array}{l}\text { Duplicados } \\
\text { Autores no identificados } \\
\text { No relacionados con el tema }\end{array}$ & $\begin{array}{l}\text { Duplicados } \\
\text { Autores no identificados } \\
\text { No relacionados con el tema }\end{array}$ \\
\hline $\begin{array}{l}\text { Número Final de } \\
\text { documentos }\end{array}$ & 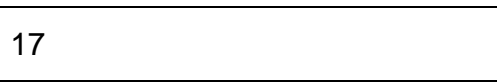 & 20 \\
\hline
\end{tabular}

Además, se han analizado documentos internos de la Universidad Rey Juan Carlos relacionados con la titulación en Administración y Dirección de Empresas, el Libro Blanco de Economía y Empresa, elaborado por una red de universidades españolas, apoyadas por la Agencia Nacional de Evaluación de la Calidad y Acreditación en España (ANECA), con el objetivo de contribuir en el diseño de los títulos de grado adaptados al Espacio Europeo de Educación Superior (EEES).

\section{OTROS ANTECEDENTES}

Existen una serie de antecedentes adicionales que es necesario detallar para documentar en mejor forma este trabajo: por un lado, se analizan las competencias que son demandadas en la industria $4.0 \mathrm{y}$, por otro, las competencias que se logran alcanzar en los estudios de grado de Administración y Dirección de Empresas de la Universidad Rey Juan Carlos.

\section{Las competencias en la Industria 4.0}

El término Industria 4.0 se utilizó por primera vez en 2011, con el objetivo de aunar las tecnologías de la información y la industria. La Industria 4.0 se basa en la adopción de tecnologías digitales para recopilar datos en tiempo real y analizarlos, proporcionando información útil al sistema de fabricación (Wang et al., 2016). El concepto surge como respuesta a la transformación de los procesos provocada por el uso de las nuevas tecnologías, Internet de las Cosas (IoT), Inteligencia Artificial (IA), minería de datos, sistemas ciber físicos (CPS), Big Data Analytics, la nube, la fabricación aditiva y las simulaciones, entre otras (Meissner et al., 2017). En otras palabras, la industria 4.0 representa la evolución de las empresas, tradicionalmente dominadas por las máquinas, hacia la digitalización, mediante la vinculación de los nuevos sistemas tecnológicos con los procesos organizativos, lo que lleva a la conexión en tiempo real entre humanos, máquinas y objetos inteligentes (Fitsilis et al., 2018).

Aunque la Industria 4.0 está generando nuevas oportunidades para las empresas, el estudio de Hecklau et al. (2016) destaca varios retos debido a la automatización y digitalización en curso. Por un lado, existen retos económicos relacionados con la creciente complejidad de los procesos resultante de la reducción del tiempo de comercialización, los ciclos de vida de los productos más cortos y los mayores niveles de personalización y flexibilidad en las expectativas de los clientes. Por otro lado, están surgiendo nuevas tendencias sociales, como la creciente importancia de la conciliación entre la vida laboral y personal y la flexibilidad laboral, así como el aumento de los puestos de trabajo que requieren una mayor cualificación y la pérdida de puestos de trabajo con menor cualificación. También hay desafíos técnicos relacionados con el tratamiento eficiente de los grandes datos, por lo que las empresas necesitan implementar amplias infraestructuras de tecnologías de la información. 
La industria 4.0 está llevando al mundo a un entorno global, automatizado, virtual y flexible (Motyl et al., 2017). Estos cambios derivan en nuevos requisitos laborales y de competencias, ya que muchos empleados trabajan hoy en día en entornos digitalizados y en red. Ello promueve la interacción con los algoritmos y la robótica, además de operar en un mundo virtual, sin embargo, uno de los mayores retos para la Industria 4.0 es la falta de cultura y competencias digitales en las organizaciones (Grzelczak et al., 2017). De acuerdo con Gitelman y Kozhevnikov (2018), los nuevos trabajadores de la industria 4.0 deberán participar en procesos complejos relacionados con las tecnologías y los mercados. Deberán tomar decisiones en un clima de incertidumbre, cambio imprevisible y riesgo considerable. De este modo, los profesionales tendrán que entender las relaciones dentro de los procesos de los cuales son responsable, los flujos de información y sus posibles interrupciones, así como las soluciones potenciales. Este mayor alcance y complejidad de la situación exigirá de los trabajadores, especialmente los gestores, una mentalidad orientada a la construcción y el mantenimiento de redes de expertos que puedan cooperar ad hoc en la búsqueda de soluciones adecuadas a problemas concretos.

Puede decirse, por tanto, que las tareas humanas se centrarán en los límites de las tareas intensivas en conocimiento, donde la flexibilidad en la resolución de problemas y la creatividad serán fundamentales para la competitividad (Erol et al., 2016). Todos los empleados a partir de un nivel intermedio de cualificación tendrán que enfrentarse a una mayor complejidad, a elementos de carácter abstracto o a la resolución de problemas a medida (Saniuk et al., 2021). En este contexto, la formación y el desarrollo profesional son fundamentales para transformar de manera significativa los perfiles laborales y de competencias de los trabajadores y así alcanzar los objetivos de la Industria 4.0 (Motyl et al., 2017). Las competencias son conjuntos de conocimientos, habilidades (técnicas y blandas) y actitudes necesarias para el desempeño eficaz de puestos de trabajo. Por un lado, los conocimientos abarcan lo que se conoce como saber qué, saber por qué, saber cómo y saber quién. Las habilidades están determinadas por la actividad práctica y la experiencia. Por último, la actitud está relacionada con la voluntad y la disposición para utilizar los conocimientos y habilidades en las acciones (Campion et al, 2011; Grzelczak et al., 2017).

El análisis de las competencias requeridas en la era de la Industria 4.0 es esencial ya que influyen en la eficiencia de los empleados, que son el capital más valioso de la empresa. Grzelczak et al. (2017) desarrollaron un modelo de competencias para hacer frente a los retos de conocimientos relacionados con las nuevas tecnologías y procesos de la Industria 4.0. Estos autores agrupan las competencias requeridas en dos categorías: técnicas y personales. De forma similar, Hecklau et al. (2016) agregan las competencias para la Industria 4.0 en cuatro grupos principales: técnicas, metodológicas, sociales y personales. Las competencias técnicas se refieren a los conocimientos y habilidades relacionados con el trabajo; las metodológicas consisten en habilidades y destrezas para la resolución de problemas generales y la toma de decisiones; las sociales incluyen habilidades y destrezas de cooperación y comunicación con los demás; y, por último, las personales están relacionadas con los valores sociales, las motivaciones y las actitudes del individuo.

La mano de obra cualificada que requiere la industria 4.0 debe poseer conocimientos y habilidades en materia de tecnologías de la información, procesamiento y análisis de datos, comprensión de la organización y los procesos, y capacidad de trabajar e interactuar con interfaces modernas. Pero requiere también de habilidades blandas, como las sociales y de comunicación y de trabajo en equipo. Además, debido a la creciente responsabilidad e influencia de los trabajadores, se necesitará capacidad de autogestión; será inevitable la confianza en las nuevas tecnologías y sistemas de asistencia y se requerirá una mentalidad general de mejora continua, dado que la dinámica de los cambios en la producción industrial será cada vez más intensa.

Gran parte de la investigación sobre las competencias requeridas para la Industria 4.0 se centra en las de naturaleza técnica (e.g. Benešová y Tupa, 2017; Pinzone et al., 2017) bajo la idea de que la introducción de las últimas tecnologías digitales y de automatización afectan significativamente a los procesos de trabajo y al entorno laboral. Sin embargo, a diferencia de las revoluciones industriales anteriores, el ser humano no puede trabajar sin la tecnología, y la tecnología no funcionará sin las personas (Grzelczak et al., 2017). Por lo tanto, la interacción con las máquinas inteligentes requiere una base de habilidades blandas o soft, como la inteligencia emocional, el pensamiento crítico, la innovación, la comunicación, la colaboración, el liderazgo y el trabajo en equipo (Wilson y Daugherty, 2018). Estas habilidades personales, sociales y metodológicas pueden aprenderse y adoptarse con el fin de lograr un equilibrio entre el conocimiento de la disciplina y las habilidades de comportamiento (Prifti et al., 2017). En este sentido, Grzelczak et al. (2017) señalan las siguientes competencias como cruciales para desarrollar la Industria 4.0 en una empresa: pensamiento y acción interdisciplinar, crecimiento del conocimiento del proceso, participación en procesos innovadores, resolución de problemas, responsabilidad personal en la toma de decisiones, habilidades sociales y comunicación, liderazgo, capacidad de coordinación de procesos de trabajo, control del ámbito de trabajo complejo y capacidad de cooperación o interacción con las máquinas. 
De manera similar, Cotet et al. (2017) afirman que para adaptarse fácilmente a los cambios incrementales propios de la naturaleza de las tecnologías de la Industria 4.0, los empleados deben desarrollar habilidades como la creatividad, la inteligencia emocional y el pensamiento proactivo. A futuro, los requisitos de habilidades se centran en la resolución de problemas complejos, el pensamiento crítico, la creatividad, la gestión de personas, la coordinación, la inteligencia emocional, el juicio y la toma de decisiones, la orientación al servicio, la negociación y la flexibilidad cognitiva.

Según Gudanowska et al. (2018), aunque existen similitudes en las competencias requeridas en diferentes industrias, estos requisitos también podrían diferir en diferentes campos. En la misma línea, Fitsilis et al. (2018) señalan que el ecosistema es complejo, ya que no existe un "determinismo tecnológico", lo que implica que tecnologías potencialmente similares pueden dar lugar a diferentes necesidades de competencias, dependiendo de los entornos organizativos. Además, podría haber diversas necesidades de competencias según los "biotopos" específicos de la Industria 4.0 y los ciclos de vida de la producción, lo que implica diferentes segmentos de mano de obra en diversos sectores que utilizan subconjuntos de tecnologías distintos.

Para abordar los aspectos anteriores, Fitsilis et al. (2018) proponen un modelo de competencias desde un enfoque multidimensional: tecnología, sector industrial, desarrollo de software y ciclo de vida de producción, habilidades transversales, competencia y perfiles de trabajo. La combinación de estas seis dimensiones debería ser capaz de proporcionar un modelo de competencias con el fin de abordar las necesidades de formación para cada caso específico o para calcular la cobertura de competencias cuando se evalúa la preparación de las empresas para introducir las tecnologías de la Industria 4.0.

La dimensión tecnológica aborda las áreas tecnológicas que están impulsando los desarrollos de las iniciativas de la Industria 4.0, como Big Data Analytics, robots autónomos, simulación, Internet de las cosas y ciberseguridad, entre otras. Dado que el software es el factor clave de las iniciativas de la Industria 4.0, la dimensión desarrollo de software y ciclo de vida de la producción se centra en la necesidad de aplicar enfoques ágiles y ajustados a la colaboración en el trabajo entre el personal de operaciones y el de desarrollo a lo largo de todas las etapas del ciclo de vida. Como resultado, se necesitan diferentes habilidades, incluyendo el análisis de negocio, el desarrollo y las pruebas de aplicaciones informáticas, la garantía de calidad, conocimiento del funcionamiento del sistema como la administración de bases de datos y redes, la gestión de sitios web, la seguridad, el control de origen, etc.

La dimensión de las competencias transversales subraya la importancia de las habilidades que pueden utilizarse en una gran variedad de situaciones y entornos de trabajo. En este sentido, deben incluirse competencias personales, sociales, relacionadas con la acción y metodológicas. La dimensión de dominio o pericia, por su parte, se refiere a la adopción de niveles de habilidad en los que los profesionales en el nivel 1 deben ser capaces de seguir o comprender un área temática, en el nivel 2 de aplicar de manera profesional una técnica de método, en el nivel 3 de diseñar soluciones o resolver problemas complejos del área temática, en el nivel 4 de dirigir equipos de profesionales y/o ofrecer consultoría en el área temática y, finalmente, en el nivel 5 de investigar o desarrollar nueva tecnología en el área temática.

Por último, la dimensión sector industrial alude a las habilidades contextuales que están relacionadas con procesos de producción específicos, normas, directrices y organización estructurada específica de un sector industrial. En la misma línea, la dimensión perfil del puesto de trabajo está relacionado con los requisitos de competencias específicas según la diversidad de funciones dentro de las empresas de la Industria 4.0, como: logística, ventas, atención al cliente, administración y gestión, mantenimiento, planificación de la producción, etc.

El trabajo de Grzybowska y Łupicka (2017) respalda las conclusiones relativas a los diferentes requisitos de competencias en función del sector y el perfil del puesto de trabajo. Este estudio se centra en el perfil laboral específico de los directivos y sugiere la existencia de diferentes necesidades de competencias técnicas, de gestión y sociales en función del sector (Benešová y Tupa, 2017; Pinzone et al., 2017; Fitsilis et al., 2018). En la Tabla 2 se recogen las competencias más relevantes para el talento de la Industria 4.0 identificadas en la literatura revisada.

\section{Las competencias en el grado ADE de la URJC}

El Espacio Europeo de Educación Superior (EEES) surge como resultado de la cooperación entre 48 países de la Unión Europea. El objetivo principal de este proyecto es fomentar el aprendizaje orientado a la adquisición de competencias con el fin de proporcionar a los estudiantes las herramientas, técnicas y habilidades que les permitieran desarrollar sus carreras profesionales. 
Tabla 2. Competencias necesarias para la Industria 4.0

\begin{tabular}{|c|c|}
\hline Categoría & Competencias \\
\hline \multirow[t]{6}{*}{ Competencias técnicas } & Conocimientos avanzados y de última generación \\
\hline & Habilidades técnicas \\
\hline & Comprensión de procesos complejos \\
\hline & $\begin{array}{l}\text { Competencias mediáticas y capacidad de utilizar medios } \\
\text { inteligentes }\end{array}$ \\
\hline & Conocimientos codificación \\
\hline & Comprensión de ciberseguridad o seguridad informática \\
\hline \multirow[t]{9}{*}{ Competencias metodológicas } & Creatividad e innovación \\
\hline & Pensamiento de acción interdisciplinar \\
\hline & Pensamiento empresaria para asumir tareas estratégicas \\
\hline & Pensamiento proactivo \\
\hline & Resolución de problemas y conflictos \\
\hline & Toma de decisiones \\
\hline & Capacidad de análisis para estructurar procesos complejos \\
\hline & Capacidad de investigación \\
\hline & Capacidad de orientación a la eficiencia \\
\hline \multirow[t]{14}{*}{ Competencias sociales } & $\begin{array}{l}\text { Habilidades interculturales } \\
\text { Comprender culturas y hábitos de trabajo diversos }\end{array}$ \\
\hline & Conocimiento de idiomas \\
\hline & Habilidades de comunicación \\
\hline & Habilidades sociales \\
\hline & Capacidad de trabajo en red \\
\hline & Capacidad de interacción con maquinas \\
\hline & Capacidad trabajo en equipo \\
\hline & Capacidad de colaboración \\
\hline & $\begin{array}{l}\text { Capacidad de compromiso y cooperación } \\
\text { Capacidad de coordinación }\end{array}$ \\
\hline & Capacidad de negociación \\
\hline & Capacidad de transferir conocimientos \\
\hline & Capacidad de orientación al servicio \\
\hline & Habilidades de Liderazgo \\
\hline & Capacidad de establecer relaciones interpersonales \\
\hline \multirow[t]{8}{*}{ Competencias personales } & Flexibilidad \\
\hline & Inteligencia emocional \\
\hline & Pensamiento crítico y tolerancia a la ambigüedad \\
\hline & Motivación para aprender \\
\hline & Capacidad para trabajar bajo presión \\
\hline & Mentalidad sostenible \\
\hline & Calidad \\
\hline & Cumplimiento y disciplina \\
\hline
\end{tabular}

El uso exclusivo de las metodologías tradicionales de enseñanza ha sido cuestionado y señalado como una aproximación errónea a la adquisición de estas competencias. Behrman y Levin (1984) expusieron que las metodologías tradicionales están enfocadas al análisis cuantitativo, las explicaciones teóricas y el uso de herramientas y modelos que permitieran la adquisición de habilidades técnicas, pero difícilmente pueden ayudar a adquirir habilidades interpersonales o de diseño. Glasser (1998) señaló que el proceso de aprendizaje no debe estar enfocado en la memorización de contenidos, dado que muchos de los conceptos serán olvidados después de la evaluación. Demostró cómo un mayor grado de participación del estudiante en la actividad de aprendizaje puede mejorar la adquisición de conocimientos y habilidades.

EI EEES ha defendido un clima de aprendizaje menos tradicional, en el que el uso de herramientas y técnicas más interactivas conlleven la participación e implicación de los estudiantes en su proceso de aprendizaje (Castaño-Perea et al., 2012), a través de un enfoque pedagógico basado en la adquisición de competencias y nuevos modelos de evaluación orientados al aprendizaje productivo (Gil-Flores, 2012). Este marco es una forma de superar planteamientos asociados a una relación jerárquica lineal entre docente y estudiante y enfocados a la memorización (López-Navas, 2014). 
La implicación de los docentes es crucial para alcanzar estos objetivos, dado que la metodología docente utilizada se convierte en una variable crítica, para determinar el grado de aprendizaje alcanzado por los estudiantes (Guardia et al., 2019). Los profesores deben evolucionar desde un rol tradicional de transmisores exclusivos del conocimiento a nuevos roles de orientadores y mentores (Wang, 2015), ayudando a los estudiantes a desarrollar su propio proceso de aprendizaje. Swartz et al. (2010), creadores de una metodología denominada Aprendizaje basado en el pensamiento, destacan la necesidad de proporcionar a los estudiantes las herramientas para afrontar los problemas y pensar de forma autónoma, que no les conduzcan simplemente a adquirir conocimiento concreto y especializado, sino competencias que les ayudarán a lo largo de toda su experiencia profesional.

Dado este contexto, las universidades deben proporcionar a los estudiantes el conocimiento, herramientas y habilidades que les capaciten para afrontar con éxito su inclusión en el entorno profesional. De forma paralela al aprendizaje de conocimientos y la adquisición de competencias para la gestión empresarial son aspectos clave de la enseñanza universitaria. Uno de los grados más implantados en las universidades españolas, dentro del EEES, es el Grado en Administración y Dirección de Empresas. Es por ello, que ha sido elegido para analizar si las competencias que se desarrollan en este grado en la Universidad Rey Juan Carlos cumplen con las demandadas por la Industria 4.0.

El Grado en Administración y Dirección de Empresas de la URJC tienen como objetivo formar profesionales capaces de desempeñar labores de gestión, asesoramiento y evaluación en las organizaciones productivas y de servicios. Estos objetivos se definen teniendo en cuenta las características y evolución de la actividad económica y empresarial en un entorno cada vez más globalizado, de rápida transformación y con mayores exigencias de competitividad y sostenibilidad. Como objetivos generales de formación se plantean conocer la naturaleza de la empresa y su relación con el entorno económico inmediato y mediato, nacional e internacional y conocer métodos y técnicas de dirección y organización de empresas y adquirir conocimientos instrumentales en matemáticas y estadística.

Las labores que los egresados tendrán que desarrollar en el mercado laboral serán en el ámbito global de la organización o en cualquiera de sus áreas funcionales: producción, recursos humanos, financiación, comercialización, inversión, administración o contabilidad. Por ello, los conocimientos a adquirir en este grado son teóricos, tanto básicos como especializados, relativos a la comprensión del funcionamiento, gestión y control de las diferentes áreas funcionales de la empresa, conocimientos socioeconómicos relativos al entorno en que se desenvuelven las empresas y conocimientos de soporte, necesarios para el correcto aprendizaje y materialización de los conocimientos anteriores.

El mercado de trabajo del área de la administración y dirección de empresas está fuertemente influido por la creación de empresas, la internacionalización, la innovación y el cambio tecnológico y las nuevas formas de organización del trabajo. Así, los diferentes perfiles profesionales que ofrece la titulación son: a) departamentos de empresas/organizaciones: financiero, comercial, control de gestión, marketing, investigación de mercados, informática, comercio internacional, compras, calidad, l+D+i, recursos humanos; b) servicios financieros: auditoría, consultoría y asesoría, mercado de valores, entidades financieras, empresas de selección, seguros, marketing, publicidad, institutos de investigación, distribución, turismo, sanitario; c) sector industrial: alimentación, bebidas, química, siderurgia, farmacéutico, automoción, papelero, cementero, editorial, cosmética, textil; d) sector infraestructuras: tecnológico, transporte, telefónico, energético, logístico, construcción; e) administración pública: internacional, europea, estatal y autonómica; f) docencia e investigación (tanto en la enseñanza secundaria como en la universidad y en el posgrado); y g) ejercicio libre de la profesión.

Por último, para que el graduado sepa identificar y anticipar oportunidades, asignar recursos, organizar la información, seleccionar y motivar a las personas, tomar decisiones, alcanzar objetivos propuestos y evaluar resultados, además de conocimientos, es necesario adquirir competencias generales y específicas que le capacite para afrontar los retos del mercado laboral. La mayoría de sus competencias, y por tanto de sus desarrollos teóricos, están fuertemente arraigados en la práctica empresarial. Si bien hay que diferenciar entre las competencias generales que los estudiantes deben adquirir para desarrollarse profesionalmente, de las competencias específicas que además de garantizar conocimientos avanzados en cada materia garanticen competencias como el compromiso ético, el aprendizaje autónomo y la capacidad de resolver problemas y tomar de decisiones, todas ellas para lograr el talento que requiere el entorno empresarial.

Las competencias generales se definen como aquellos atributos que debe tener cualquier graduado universitario con independencia de su titulación. En este primer grupo, se deberán recoger los conocimientos, habilidades, destrezas y capacidades que debe tener todo titulado/a para incorporarse al mercado laboral. Las competencias genéricas han sido recogidas por ANECA en el Libro Blanco, a partir de la lista sugerida por el Proyecto Tuning, que las organiza en los siguientes grupos: a.1) competencias instrumentales: que proporcionan herramientas para el desarrollo de la actividad profesional; a.2) competencias personales: capacidades individuales relativas a la expresión de los propios planteamientos, habilidades críticas y de 
autocrítica. Se relacionan con destrezas de índole social, con las habilidades interpersonales de los individuos, la capacidad de trabajar en equipo o la expresión de compromiso social o ético. Estas competencias tienden a facilitar los procesos de interacción social y cooperación; a.3) competencias sistémicas: son las destrezas y habilidades que conciernen a los sistemas como totalidad. Suponen una combinación de la comprensión, la sensibilidad y el conocimiento que permiten al individuo ver cómo las partes de un todo se relacionan y se agrupan. Estas capacidades incluyen la habilidad de planificar los cambios, de manera que puedan hacerse mejoras en los sistemas y rediseñarlos. Las competencias sistémicas o integradoras requieren como base la adquisición previa de competencias instrumentales y personales; a.4) las competencias prácticas (transferibles) permiten cubrir la brecha entre la teoría y la realidad, es decir, entre la teoría y el mercado laboral.

Por su parte, las competencias específicas se relacionan con cada una de las áreas temáticas. Son fundamentales en la definición de las titulaciones porque están directamente vinculadas con sus propias materias. Confieren identidad y consistencia al programa de Grado, y pueden ser clasificadas en dos grandes grupos: b.1) conocimientos básicos: relacionadas con la formación académica que deben adquirir los graduados, es decir, el "saber" que ha de transmitirse a los estudiantes a través de las distintas materias recogidas en el programa. La relación de conocimientos básicos propuestos se corresponde con las materias de formación básica acordadas para todas las titulaciones de la Rama de Ciencias Económicas y Empresariales; b.2) competencias profesionales: asociadas a las habilidades, destrezas y conocimientos prácticos que han de ser aprendidos durante la estancia del estudiante en la universidad para el desarrollo de una profesión.

Es importante destacar que tanto las competencias generales como las específicas de los Grados se van actualizando con el paso del tiempo para adaptarse al contexto empresarial y educativo. El proceso de actualización comienza con los docentes que son quiénes en contacto directo con los estudiantes y la realidad de la asignatura que se imparte detectan las modificaciones necesarias de las diferentes asignaturas del itinerario formativo para adaptarse a la nueva realidad empresarial. Los docentes, coordinados entre ellos, cada año pueden sugerir a los responsables de asignaturas que incluyan o eliminen competencias en las guías docentes. Si el responsable de asignatura lo considera necesario, solicita a los coordinadores de grado la actualización de la guía docente en función de las mejoras detectadas. A su vez, los coordinadores de grado elevan esta solitud al Vicerrectorado de Calidad que es el estamento responsable, en último término, de modificar las memorias de los grados de la universidad. Estas mejoras del grado se envían, para su estudio, a la Agencia Nacional de Evaluación de la Calidad y Acreditación (ANECA). Por último, toda la información sobre la memoria y competencias de los grados es publicada en el Registro de Universidades, Centros y Títulos (RUCT) perteneciente al Ministerio de Ciencia, Innovación y Universidades del Gobierno de España.

Las competencias genéricas y específicas del Grado de Administración y Dirección de Empresas están específicamente identificadas en la web de la Universidad Rey Juan Carlos, de forma que la accesibilidad a esta información es fácil para coordinadores, docentes y estudiantado. A continuación, se recogen, de forma detallada, en la Tabla 3. Una de las herramientas implantadas por el EEES, es la obligatoriedad de que los estudiantes en el último curso del grado en Administración y Dirección de Empresas completen su formación en la universidad, con prácticas externas desarrolladas en empresas. La asignatura de prácticas externas son 24 créditos (500 horas) del total de 240 créditos que computan el Grado completo. Algunas de las empresas que tienen acuerdos de colaboración con la URJC para llevar a cabo estas prácticas son Banco Santander, Repsol, Mapfre, 3M España, Laboratorios Cinfa, Ferrovial, Atos IT o Schindler.

Analizando la guía docente de la asignatura Prácticas Externas se indica que esta formación constituye una obligación jurídica de primer orden, correlativa al derecho de los estudiantes a su realización y desarrollo, conforme el artículo 24 del Real Decreto 1791/2010, de 30 de diciembre, en las condiciones que la Universidad establezca, y el propio artículo 141 a) de los Estatutos de la Universidad Rey Juan Carlos, en cuanto al derecho de los mismos a recibir una formación integral y una enseñanza de calidad, tanto teórica como práctica.

Mediante la realización de las prácticas externas, los estudiantes de la Universidad Rey Juan Carlos se inician en el ejercicio de actividades profesionales relacionadas, principalmente con su formación, preparándose de ese modo, para su incorporación al mercado de trabajo, como finalidad primordial. El objetivo de la asignatura es proporcionar la formación académica necesaria para preparar al estudiante en el ejercicio de actividades profesionales y facilitar así su incorporación al mercado de trabajo y se destacan las siguientes competencias a adquirir: 1) capacidad de análisis y síntesis: analizar, sintetizar, valorar y tomar decisiones a partir de los registros relevantes de información sobre la situación y previsible evolución de una empresa; 2) capacidad de organización y planificación: organizar, planificar y administrar una empresa u organización de tamaño pequeño y mediano, entendiendo su ubicación competitiva e institucional e identificando sus fortalezas y debilidades; 3) capacidad para trabajar en equipo; 4) capacidad para trabajar en entornos diversos y multiculturales: reconocimiento y respeto a la diversidad; 5) liderazgo; y 6) aplicar los conocimientos en la práctica. 
Tabla 3. Competencias generales y específicas del Grado de Administración y Dirección de Empresas

\begin{tabular}{|c|c|c|}
\hline \multicolumn{3}{|c|}{ Competencias Generales } \\
\hline $\begin{array}{l}\text { Competencias } \\
\text { Instrumentales }\end{array}$ & \multicolumn{2}{|c|}{$\begin{array}{l}\text { Cl1 Capacidad análisis y síntesis a partir de registros relevantes de información sobre la } \\
\text { situación y previsible evolución de una empresa. } \\
\text { Cl2 Capacidad organización y planificación de una empresa u organización de tamaño } \\
\text { pequeño y mediano, entendiendo su ubicación competitiva e institucional e identificando sus } \\
\text { fortalezas y debilidades. } \\
\text { Cl3 Comunicación en lenguaje nativo: comprender y ser capaz de comunicarse, con } \\
\text { corrección y en diferentes escenarios. Elaborar informes de asesoramiento y proyectos de } \\
\text { Gestión empresarial. } \\
\text { Cl4 Comunicación en una lengua extranjera (inglés). } \\
\text { Cl5 Conocimientos de informática en todo su desempeño profesional. } \\
\text { Cl6 Capacidad Gestión de la información: analizar, buscar y discriminar información } \\
\text { proveniente de fuentes diversas. } \\
\text { Cl7 Capacidad para la resolución de problemas. } \\
\text { Cl8 Capacidad de tomar decisiones. }\end{array}$} \\
\hline $\begin{array}{l}\text { Competencias } \\
\text { Personales }\end{array}$ & $\begin{array}{l}\text { CP1 Capacidad para trabajar en equipo } \\
\text { CP2 Trabajo en un contexto } \\
\text { internacional. } \\
\text { CP3 Capacidad trabajar en entornos } \\
\text { diversos y multiculturales. }\end{array}$ & $\begin{array}{l}\text { CP4 Capacidad de Razonamiento crítico. } \\
\text { CP5 Compromiso ético en el trabajo. }\end{array}$ \\
\hline $\begin{array}{l}\text { Competencias } \\
\text { Sistémicas }\end{array}$ & $\begin{array}{l}\text { CS1 Aprendizaje autónomo. } \\
\text { CS2 Adaptación a nuevas situaciones. } \\
\text { CS3 Creatividad. } \\
\text { CS4 Liderazgo. }\end{array}$ & $\begin{array}{l}\text { CS5 Iniciativa y espíritu emprendedor. } \\
\text { CS6 Motivación por la calidad. } \\
\text { CS7 Comprender responsabilidad social } \\
\text { derivada de las actuaciones económicas y } \\
\text { empresariales }\end{array}$ \\
\hline $\begin{array}{l}\text { Competencias } \\
\text { Prácticas }\end{array}$ & \multicolumn{2}{|c|}{$\begin{array}{l}\text { CPR1 Aplicar los conocimientos en la práctica. } \\
\text { CPR2 Capacidad para la investigación. }\end{array}$} \\
\hline \multicolumn{3}{|c|}{ Competencias Específicas } \\
\hline $\begin{array}{l}\text { Conocimientos } \\
\text { Básicos }\end{array}$ & $\begin{array}{l}\text { CE1 Organización de Empresas } \\
\text { CE2 Marketing } \\
\text { CE3 Contabilidad } \\
\text { CE4 Finanzas } \\
\text { CE5 Recursos Humanos } \\
\text { CE6 Economía } \\
\text { CE7 Derecho }\end{array}$ & $\begin{array}{l}\text { CE8 Sociología } \\
\text { CE9 Historia } \\
\text { CE10 Matemáticas } \\
\text { CE11 Estadística } \\
\text { CE12 Tecnologías de la información } \\
\text { CE13 Idioma }\end{array}$ \\
\hline $\begin{array}{l}\text { Competencias } \\
\text { Profesionales }\end{array}$ & \multicolumn{2}{|c|}{$\begin{array}{l}\text { CP1 Dominio herramientas informáticas aplicadas con carácter específico a las diferentes } \\
\text { materias } \\
\text { CP2 Capacidad aplicación conocimientos teóricos, metodológicos y técnicas adquiridas. } \\
\text { CP4 Capacidad utilizar herramientas cuantitativas en la toma de decisiones empresariales } \\
\text { CP3 Capacidad para modelizar situaciones empresariales } \\
\text { CP5 Capacidad para analizar y diseñar la estructura organizativa } \\
\text { CP6 Visión estratégica de la dirección de empresas } \\
\text { CP7 Capacidad para gestionar recursos humanos } \\
\text { CP8 Capacidad para dirigir las operaciones y organizar la producción } \\
\text { CP9 Capacidad determinar indicadores y agregados económicos y su impacto en la empresa } \\
\text { CP10 Habilidad para interpretar el contexto económico nacional e internacional. } \\
\text { CP11 Capacidad para elaborar e interpretar la información contable } \\
\text { CP12 Comprensión de las operaciones financieras que tienen lugar en el ámbito empresarial } \\
\text { CP13 Capacidad para resolver problemas de valoración financiera e inversión empresarial } \\
\text { CP14 Dominio de las herramientas informáticas aplicadas a Gestión financiera-contable } \\
\text { CP15 Capacidad para diseñar e implantar las estrategias de marketing } \\
\text { CP16 Capacidad evaluar mercado y entorno integrado del sistema de información del } \\
\text { marketing } \\
\text { CP17 Capacidad para comprender las implicaciones internacionales de la Gestión } \\
\text { empresarial } \\
\text { CP18 Capacidad para aplicar conceptos básicos de Derecho } \\
\text { CP19 Comprensión marco histórico de referencia: ubicar la problemática objeto de estudio } \\
\text { CP20 Capacidad para identificar y cuantificar la incertidumbre en problemas de decisión } \\
\text { CP21 Capacidad aplicar el lenguaje matemático/estadístico en problemas económico- } \\
\text { empresarial }\end{array}$} \\
\hline
\end{tabular}


Para evaluar la adquisición de estas competencias se utiliza el criterio de ponderar tres aspectos: $60 \%$ el informe final del tutor de la empresa, $20 \%$ el informe final del tutor académico y $20 \%$ memoria final entregada por el estudiante. Adicionalmente, se ha realizado el análisis de las competencias recogidas en las guías docentes del resto de las asignaturas del Administración y Dirección de Empresas. A través de este estudio se pretende hacer un análisis comparado: competencias demandadas en la Industria 4.0 versus las competencias ofertadas a los estudiantes del grado de Administración de Empresas y sus dobles grados asociados. Con ello se pretende detectar aquellas competencias del talento que necesita la Industria 4.0 que no se estén aportando a los futuros profesionales de dicha industria.

\section{RESULTADOS Y DISCUSIÓN}

Las competencias generales y específicas del grado de administración y dirección de empresas, y sus dobles grados asociados, se pueden asimilar a las competencias directivas propuestas por Katz (1974). Así, las competencias específicas se reflejan en las habilidades técnicas, que comprenden el conocimiento especializado en torno a actividades específicas, así como la capacidad de analizar problemas relacionados con el área de especialización y la capacidad para utilizar técnicas y herramientas vinculadas a una disciplina concreta. Por su parte, las habilidades humanas o interpersonales, relacionadas con el trabajo en equipo y la capacidad para aglutinar los esfuerzos colectivos de cara a la consecución de un objetivo se ven reflejadas, en gran medida, en las competencias genéricas personales.

Por último, las habilidades conceptuales, que permiten a un directivo desarrollar una visión holística de la empresa que le otorgue la capacidad de entender las relaciones entre las diferentes áreas funcionales y unidades de negocio de la compañía y los impactos cruzados de las actuaciones desarrolladas en una de ellas, se reflejan claramente en las competencias sistémicas. Koontz y Weihrich (2017) definieron un cuarto tipo, las habilidades de diseño, que hacen referencia a la capacidad para diagnosticar problemas y proponer soluciones. Estos grupos de habilidades de gestión pueden relacionarse de forma relativamente simple con los roles directivos planteados por Mintzberg (1990). Estas competencias se reflejan en las competencias generales prácticas y las competencias específicas profesionales. Una vez comparadas las competencias necesarias de la Industria 4.0 identificadas en la revisión de la literatura con las competencias recogidas en las guías docentes de las asignaturas de $A D E$, en la Tabla 4 se recogen las correlaciones y similitudes encontradas entre las competencias demandadas y las competencias ofertadas.

Tabla 4. Competencias necesarias para la Industria 4.0 vs Competencias del Grado ADE

\begin{tabular}{|c|c|c|}
\hline Categoría & $\begin{array}{l}\text { Competencias } \\
\text { necesarias Industria } 4.0\end{array}$ & $\begin{array}{l}\text { Competencias ofertadas } \\
\text { Grado } A D E\end{array}$ \\
\hline \multirow{6}{*}{ 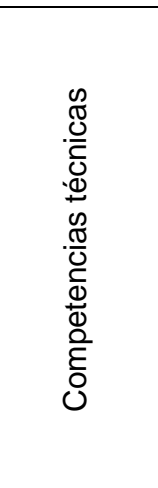 } & $\begin{array}{l}\text { Conocimientos última } \\
\text { generación }\end{array}$ & $\begin{array}{l}\text { Competencia específica del conocimiento en cada asignatura } \\
\text { Conocimiento de informática relativo al ámbito de estudio }\end{array}$ \\
\hline & Habilidades técnicas & $\begin{array}{l}\text { Dominio de herramientas informáticas aplicadas con carácter } \\
\text { específico a las diferentes materias }\end{array}$ \\
\hline & $\begin{array}{l}\text { Comprensión del } \\
\text { proceso }\end{array}$ & Capacidad de gestión de la información: analizar, buscar y discriminar \\
\hline & $\begin{array}{l}\text { Competencias } \\
\text { mediáticas }\end{array}$ & Tecnologías de la información \\
\hline & $\begin{array}{l}\text { Conocimientos } \\
\text { codificación }\end{array}$ & Capacidad de gestión de la información: analizar, buscar y discriminar \\
\hline & $\begin{array}{l}\text { Comprensión seguridad } \\
\text { informática }\end{array}$ & No se oferta \\
\hline \multirow{8}{*}{ 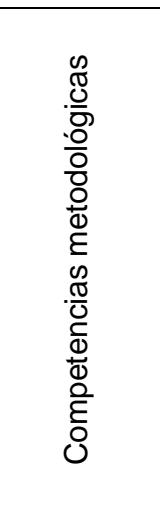 } & Creatividad & No se oferta \\
\hline & $\begin{array}{l}\text { Pensamiento } \\
\text { empresarial }\end{array}$ & Capacidad de organización y planificación \\
\hline & Resolución de problemas & Capacidad para la resolución de problemas \\
\hline & Resolución de conflictos & Capacidad para la resolución de problemas \\
\hline & Toma decisiones & Capacidad para tomar decisiones \\
\hline & Capacidad análisis & Capacidad de análisis y síntesis \\
\hline & $\begin{array}{l}\text { Capacidad de } \\
\text { investigación }\end{array}$ & $\begin{array}{l}\text { Capacidad para identificar y cuantificar la incertidumbre en problemas } \\
\text { de decisión }\end{array}$ \\
\hline & Orientación eficiencia & $\begin{array}{l}\text { Capacidad para resolver problemas de decisiones de financiación e } \\
\text { inversión empresarial } \\
\text { Motivación por la calidad }\end{array}$ \\
\hline
\end{tabular}


Tabla 4 (continuación)

\begin{tabular}{|c|c|c|}
\hline Categoría & $\begin{array}{l}\text { Competencias } \\
\text { necesarias Industria } 4.0\end{array}$ & $\begin{array}{l}\text { Competencias ofertadas } \\
\text { Grado ADE }\end{array}$ \\
\hline \multirow{8}{*}{ 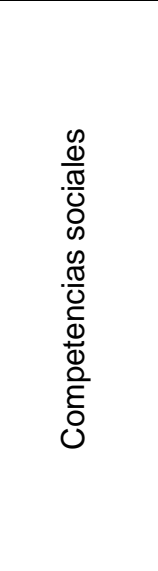 } & $\begin{array}{l}\text { Habilidades } \\
\text { interculturales }\end{array}$ & Capacidad para trabajar en entornos diversos y multiculturales \\
\hline & Conocimiento idiomas & $\begin{array}{l}\text { Conocimiento de una segunda lengua } \\
\text { Capacidad de trabajar en entornos multilingües y multidisciplinares }\end{array}$ \\
\hline & $\begin{array}{l}\text { Habilidades } \\
\text { comunicación }\end{array}$ & $\begin{array}{l}\text { Capacidad de comprender y ser capaz de comunicarse con corrección } \\
\text { y en diferentes escenarios }\end{array}$ \\
\hline & Capacidad trabajo en red & Capacidad para trabajar en equipo \\
\hline & $\begin{array}{l}\text { Capacidad trabajo en } \\
\text { equipo }\end{array}$ & Capacidad para trabajar en equipo \\
\hline & $\begin{array}{l}\text { Capacidad compromiso y } \\
\text { cooperación }\end{array}$ & Compromiso ético en el trabajo \\
\hline & $\begin{array}{l}\text { Capacidad transferir } \\
\text { conocimientos }\end{array}$ & Aplicar los conocimientos en la práctica \\
\hline & Habilidades de liderazgo & Liderazgo \\
\hline \multirow{6}{*}{ 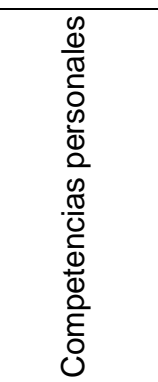 } & Flexibilidad & No se oferta \\
\hline & $\begin{array}{l}\text { Tolerancia a la } \\
\text { ambigüedad }\end{array}$ & No se oferta \\
\hline & $\begin{array}{l}\text { Motivación para } \\
\text { aprender }\end{array}$ & Aprendizaje autónomo \\
\hline & $\begin{array}{l}\text { Capacidad para trabajar } \\
\text { bajo presión }\end{array}$ & No se oferta \\
\hline & Mentalidad sostenible & No se oferta \\
\hline & Cumplimiento & Compromiso ético en el trabajo \\
\hline
\end{tabular}

Analizadas todas las competencias se concluye que algunas de las competencias demandadas por la Industria 4.0 claramente se recogen entre las ofertadas en el grado de administración y dirección de empresas de la URJC, otras aunque se identifican, no son nombradas de igual forma y algunas no quedan expresamente recogidas. Nuestro análisis comparado revela que las competencias técnicas que se demandan en la Industria 4.0 quedan ampliamente recogidas entre las competencias específicas del grado ADE tanto si se refieren a los conocimientos básicos como a las competencias profesionales. Las competencias metodológicas y las competencias sociales se recogen dentro del conjunto de las competencias generales clasificadas como instrumentales, sistémicas y prácticas. Sin embargo, aunque en el grado de ADE de la URJC se ofertan competencias personales, estas no recogen todas las competencias personales que demanda la Industria 4.0. Las relaciones entre las competencias demandadas en la Industria 4.0 y las ofertadas en el grado ADE de la URJC se representan en la Figura 1.

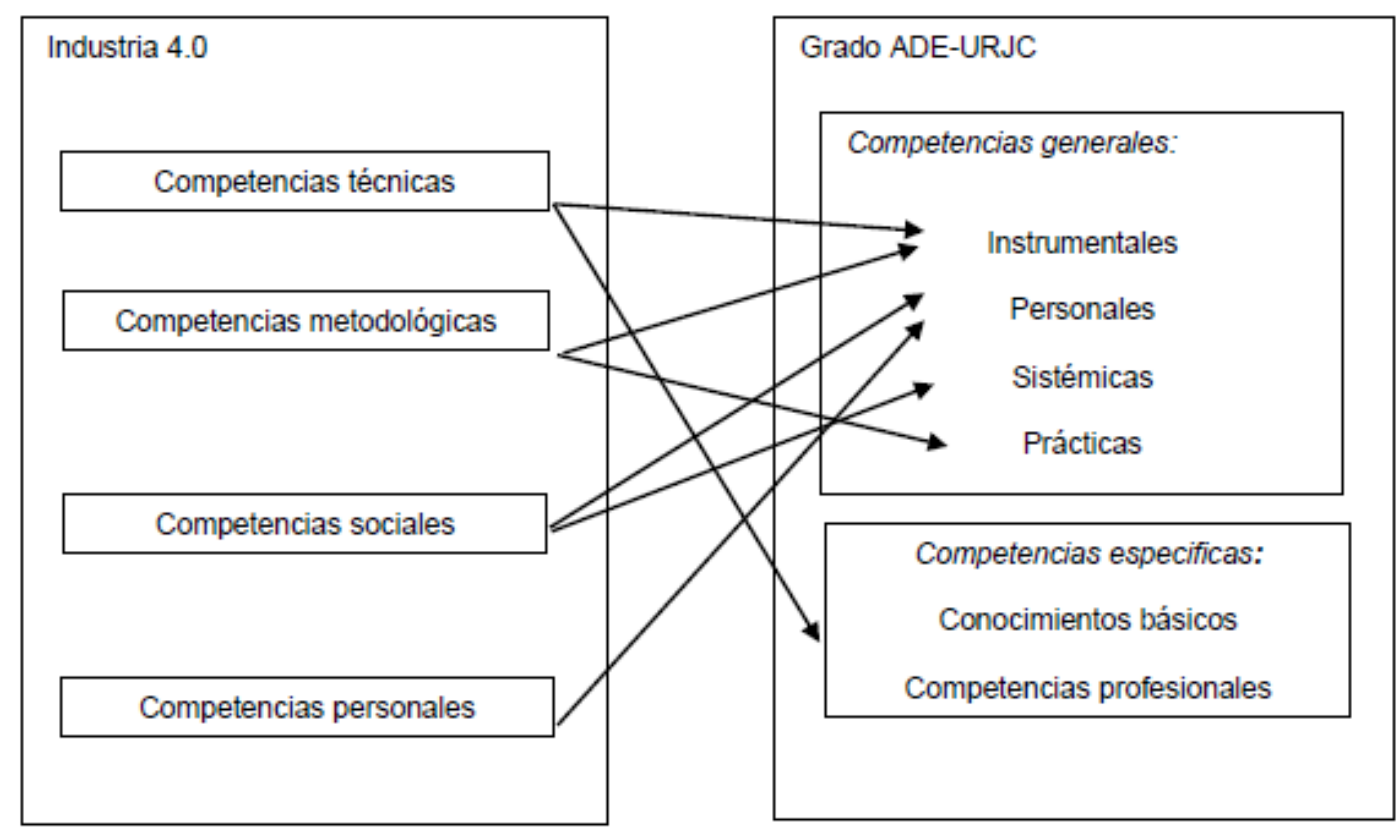

Fig. 1. Análisis comparado competencias demandadas vs ofertadas 
Las competencias demandadas por la Industria 4.0 que no se recogen en el grado ADE son: una competencia técnica (comprensión de la seguridad Informática: ciberseguridad); una competencia metodológica (creatividad); y cuatro competencias personales (flexibilidad, tolerancia a la ambigüedad, capacidad para trabajar bajo presión y mentalidad sostenible). De entre ellas, la creatividad y flexibilidad si bien es cierto que no quedan explícitamente recogidas como competencias generales o específicas, son factores que se desarrollan en profundidad dentro del itinerario formativo de este grado. Las competencias metodológicas son esenciales en este nuevo entorno ya que la seguridad informática, es un aspecto básico en organizaciones de cualquier tamaño y sector que invierten fuertemente en tecnología de ciberdefensa con el fin de proteger sus activos críticos: marca, capital intelectual y la información de sus clientes. Se trata de un aspecto que tiene que ver con la protección de datos contra accesos no autorizados y para protegerlos de una posible corrupción durante todo su ciclo de vida. Incluye conceptos como encriptación de datos, tokenización y prácticas de gestión de claves que ayudan a proteger los datos en todas las aplicaciones y plataformas de una organización.

Respecto a las competencias personales, en la Industria 4.0 se requiere que los individuos tengan capacidad creativa para aportar ideas nuevas y originales y también capacidad innovadora para convertir las ideas en productos, procesos y servicios nuevos y mejorados que el mercado reconozca y valore. Además, se requiere ser flexibles y tener la capacidad para poder adaptarse a los continuos cambios que se produzcan en la organización (económicos, tecnológicos, sociales o culturales), con el objetivo de mantener o mejorar la posición competitiva dentro del mercado laboral. Respecto a las competencias personales, la tolerancia a la ambigüedad se refiere a la capacidad para permanecer algún tiempo en situaciones confusas y no resueltas sin precipitarse por resolverlas forzando un cierre prematuro de la situación problemática. Tolerar no implica permanecer en la ambigüedad, y tampoco apunta a una experiencia caótica, indiscriminada, sino que incluye una forma de ir asimilando la experiencia de manera ordenada sin forzar las respuestas. La capacidad para trabajar bajo presión se refiere a la capacidad de desarrollar determinadas tareas profesionales bajo condiciones adversas tanto de tiempo como de sobrecarga, manteniendo un ritmo de eficiencia y eficacia. $Y$ la Mentalidad sostenible supone la responsabilidad personal sobre lo que ocurrirá en el futuro, como producto las propias decisiones presentes, cautelando y actuando sobre la tridimensionalidad de la sostenibilidad.

\section{DISCUSION FINAL}

La Industria 4.0 representa un entorno global, automatizado, virtual y flexible que exige nuevas competencias y habilidades en el entorno laboral. Por ello, se hace necesario abordar estas cuestiones desde el ámbito de la educación universitaria, donde las personas se capacitan para insertarse en el mundo laboral. Con este estudio se ha realizado un análisis comparativo entre las competencias que se demandan en la Industria 4.0 y las competencias que la Universidad Rey Juan Carlos establece en los itinerarios formativos del plan de estudio del grado de Administración y Dirección de Empresas y sus dobles grados asociados.

Como primera contribución, la literatura revela que los modelos de competencias en la era de la Industria 4.0 se agrupan en torno a cuatro categorías principales: técnicas, metodológicas, sociales y personales. A pesar de la importancia de las habilidades técnicas para cualquier revolución industrial, en el caso que nos ocupa cobran especial importancia las competencias de naturaleza personal, social y metodológica para alcanzar un equilibrio entre el conocimiento de la disciplina y las habilidades de comportamiento. Cabe también destacar que estas capacidades no son homogéneas para toda la industria 4.0, sino que tecnologías potencialmente similares pueden dar lugar a diferentes necesidades de competencias en función de los entornos organizativos o los ciclos de vida de la producción. Por tanto, se debe tener en cuenta las particularidades de cada empresa a la hora de diseñar los diferentes modelos competenciales.

Como segunda contribución, se detecta que el modelo de competencias del Grado de Administración y Dirección de Empresas organiza las habilidades en función de la especificidad de las mismas. De este modo, se destacan las competencias específicas -relacionadas tanto con los conocimientos o formación académica en distintas materias o asignaturas como la contabilidad, las finanzas o los recursos humanos-, así como las competencias profesionales asociadas a las habilidades, destrezas y conocimientos prácticos. Por otro lado, el resto de las competencias necesarias para desarrollarse profesionalmente se engloban dentro de la categoría "generales", donde podemos identificar cuatro categorías: instrumentales, personales, sistémicas y prácticas.

Finalmente, como última contribución se han comparado las exigencias de la Industria 4.0 versus la realidad del marco competencial del Grado de Administración y Dirección de Empresas. De este modo, se ha estudiado que existen competencias técnicas, metodológicas y personales que no están recogidas. Se considera que hay que hacer una profunda reflexión para incorporarlas porque son clave en el entorno actual. Es cierto que, si bien la creatividad y la flexibilidad no quedan explícitamente recogidas como competencias generales o 
específicas del grado de administración y dirección de empresas, son factores que se desarrollan en profundidad dentro del itinerario formativo. Son aspectos a los que los docentes de este grado insisten en desarrollar tanto en las asignaturas de dirección estratégica como en las de recursos humanos: ser flexibles y creativos son factores fundamentales del entorno y se les explica y exige a los alumnos. Hay otras dos competencias: tolerancia a la ambigüedad y capacidad para trabajar bajo presión que, aunque se tratan de incorporar con trabajos en grupos diversos y planteando retos, quizás no se logre al nivel que exige la Industria 4.0. Por su parte, las dos últimas, ciberseguridad y mentalidad sostenible, se considera que son esenciales y no se recogen en las guías docentes.

\section{CONCLUSIONES}

De acuerdo al trabajo presentado y a los resultados obtenidos, se pueden plantear las siguientes conclusiones principales, que dan respuesta a las preguntas de investigación planteadas:

1.- La Industria 4.0, por su elevado carácter tecnológico basada en la digitalización y también en la sostenibilidad reclama una serie de competencias específicas como son la capacidad de trabajar bajo presión, la capacidad de ser flexibles y de ser tolerantes a la ambigüedad, así como la conciencia por la ciberseguridad y la sostenibilidad.

2.- Las competencias que se pretenden lograr entre los alumnos egresados en el grado de administración y dirección de empresas de la Universidad Rey Juan Carlos son clasificadas en específicas y generales donde se identifican cuatro categorías: instrumentales, personales, sistémicas y prácticas y que son ampliamente convergentes con las reclamadas en la Industria 4.0

3.- Sin embargo, se han detectado que las competencias demandadas: creatividad, flexibilidad, tolerancia a la ambigüedad, capacidad de trabajar bajo presión, ciberseguridad y mentalidad sostenible no están específicamente recogidas en las guías docentes de las asignaturas que componen el itinerario formativo del grado de administración y dirección de empresas de la URJC.

El resultado de este estudio indica que estas competencias esenciales para la Industria 4.0 se deben incluir entre las competencias genéricas y específicas de los estudios de administración y dirección de empresas, y sus dobles grados asociados de la Universidad Rey Juan Carlos. Una vez realizado el diagnóstico de la situación de las competencias demandas vs ofertadas, se requieren análisis empíricos que contrasten las competencias demandadas en el nuevo entorno con las ofertadas en la formación universitaria tomando como referente información del desempeño de los estudiantes en sus prácticas externas, los procesos de autoevaluación institucional, así como de los docentes a cargo del proceso formativo, y todo ello teniendo en cuenta la percepción del estudiantado.

\section{REFERENCIAS}

Behrman, J., y Levin, R., Are Business Schools doing their job?, Harvard Business Review, ISSN 0017-8012, 62(1), 140147 (1984)

Benešová, A., y Tupa, J., Requirements for education and qualification of people in Industry 4.0, https://doi.org/10.1016/j.promfg.2017.07.366, Procedia Manuf., 11, 2195-2202 (2017)

Campion, M.A., Fink, A.A., y otros 4 autores, Doing competencies well: Best practices in competency modeling, https://doi.org/10.1111/j.1744-6570.2010.01207.x, Pers. Psychol., 64(1), 225-262 (2011)

Castaño-Perea, E., Fernández, A. B., y Castañeda, E. A., Competencias para la tutoría: experiencia de formación con profesores universitarios, REDU: Revista de Docencia Universitaria, ISSN 1887-4592,10(2), 193-210 (2012)

Cotet, G.B., Balgiu, B.A., y Zaleschi, V.C., Assessment procedure for the soft skills requested by Industry 4.0, https://doi.org/10.1051/matecconf/201712107005, MATEC Web of Conferences, 121(07005), 1-8 (2017)

Chryssolouris, G., Mavrikios, D., y Mourtzis, D., Manufacturing systems: skills \& competencies for the future, https://doi.org/10.1016/j.procir.2013.05.004, Procedia CIRp, 7, 17-24 (2013)

Erol, S., Jäger, A., y otros 3 autores, Tangible Industry 4.0: a scenario-based approach to learning for the future of production, https://doi.org/10.1016/j.procir.2016.03.162, Procedia CIRP 54, 13-18 (2016)

Fitsilis, P., Tsoutsa, P., y Gerogiannis, V., Industry 4.0: Required personnel competences, Industry 4.0, ISSN 2534-8582, 3(3), 130-133 (2018)

Gehrke, L., Kühn, A.T., y otros 5 autores, A discussion of qualifications and skills in the factory of the future: A German and American perspective, $1^{\mathrm{a}}$ Ed., 1-28, VDI/ASME Industry, Dusseldorf, Alemania (2015)

Gitelman, L. D., y Kozhevnikov, M. V., Paradigm of managerial education for a technological breakthrough in the economy, https://doi.org/10.17059/2018-2-8, Economy of Region, 1(2), 433-449 (2018) 
Gil-Flores, J., La evaluación del aprendizaje en la universidad según la experiencia de los estudiantes, Estudios sobre Educación, ISSN 1578-7001, 22, 133- 153 (2012)

Glasser, W., Choice Theory: A new psychology of personal freedom, $1^{\text {a } E d ., ~ 25-43, ~ H a r p e r ~ C o l l i n s, ~ N u e v a ~ Y o r k, ~ E s t a d o s ~}$ Unidos (1998)

Goulart, V. G., Liboni, L. B., y Cezarino, L. O., Balancing skills in the digital transformation era: The future of jobs and the role of higher education, https://doi.org/10.1177/09504222211029796, Industry and Higher Education, 1-10 (2021)

Grzelczak, A., Kosacka, M., y Werner-Lewandowska, K., Employees competences for Industry 4.0 in Poland-preliminary research results, 24th International Conference on Production Research (ICPR), 139-144, Poznan, Polonia (2017)

Grzybowska, K., y Łupicka, A., Key competencies for Industry 4.0, Economics \& Management Innovations, 1(1), 250253, (2017)

Guardia, J., Del Olmo, J., Roa, I., y, Berlanga, V., Innovation in the teaching-learning process: the case of Kahoot!, https://doi.org/10.1108/OTH-11-2018-0035, On the Horizon, 27(1), 35-45 (2019)

Gudanowska, A. E., Alonso, J. P., y Törmänen, A., What competencies are needed in the production industry? The case of the Podlaskie Region, https://doi.org/10.1515/emj-2018-0006, Engineering Management in Production and Services, 10(1), 65-74 (2018)

Hecklau, F., Galeitzke, M., Flachs, S., y Kohl, H., Holistic approach for human resource management in Industry 4.0, https://doi.org/10.1016/j.procir.2016.05.102, Procedia CIRp, 54, 1-6 (2016)

Katz, R., Skills of an effective administrator, Harvard Business Review, ISSN 0017-8012, 52(5), 90-102 (1974)

Koontz, H., y Weihrich, H., Administración: una perspectiva Global, 15aㅡd., 1-646, McGraw Hill, México D.F. México (2017)

López-Navas, C., Educación superior y TIC: conceptos y tendencias de cambio, https://doi.org/10.5209/rev_HICS.2014.v19.45128, Historia y Comunicación Social, 19, 227-239 (2014)

Maisiri, W., Darwish, H., y van Dyk, L., An investigation of industry 4.0 skills requirements, http://dx.doi.org/10.7166/30-32230, S. Afr. J. Ind. Eng., 30(3), 90-105 (2019)

Meissner, H., Ilsen, R., y Aurich, J.C., Analysis of control architectures in the context of Industry 4.0, https://doi.org/10.1016/j.procir.2016.06.113, Procedia CIRp, 62, 165-169 (2017)

Mintzberg, H., The manager's job, Folklore and Fact, Harvard Business Review, ISSN 0017-8012, 68(2), 163-176 (1990)

Moldovan, L., State-of-the-art Analysis on the Knowledge and Skills Gaps on the Topic of Industry 4.0 and the Requirements for Work-based Learning, https://doi.org/10.1016/j.promfg.2019.02.217, Procedia Manuf., 32, 294-301 (2019)

Motyl, B., Baronio, G., y otros 3 autores, How will change the future engineers' skills in the Industry 4.0 framework? A questionnaire survey, https://doi.org/10.1016/j.promfg.2017.07.282, Procedia Manuf., 11, 1501-1509 (2017)

Pinzone, M., Fantini, P., y otros cuatro autores, Jobs and skills in Industry 4.0: an exploratory research", IFIP International Conference on Advances in Production Management Systems, 282-288, Hamburgo, Alemania (2017)

Prifti, L., Knigge, M., Kienegger, H., y Krcmar, H., A Competency Model for "Industrie 4.0" Employees, 13th Internationalen Tagung Wirtschaftsinformatik, 46-60, St. Gallen, Alemania (2017)

Rivera, F. C., Hermosilla, P., Delgadillo, J., y Echeverría, D., Propuesta de construcción de competencias de innovación en la formación de ingenieros en el contexto de la industria 4.0 y los objetivos de desarrollo sostenible (ODS), http://dx.doi.org/10.4067/S0718-50062021000200075, Formación Universitaria, 14(2), 75-84 (2021)

Saniuk, S., Caganova, D., y Saniuk, A., Knowledge and Skills of Industrial Employees and Managerial Staff for the Industry 4.0 Implementation, https://doi.org/10.1007/s11036-021-01788-4, Mobile Networks and Applications, 1-11 (2021)

Schuh, G., Potente, T., y otros 3 autores, Collaboration moves productivity to the next level, https://doi.org/10.1016/j.procir.2014.02.037, Procedia CIRp, 17, 3-8 (2014)

Swartz, R. J., Costa, A. L., y otros 3 autores, Thinking-Based Learning: Promoting Quality Student Achievement in the 21st Century, $1^{\text {a }}$ Ed., 1-288, Teachers College Press, Amsterdam, Países Bajos (2010)

Wang, A., The wear out effect of a game-based student response system,

https://doi.org/10.1016/j.compedu.2014.11.004, Computers \& Education, 82, 217-227 (2015)

Wang, S., Wan, J., Li, D., y Zhang, C., Implementing smart factory of industrie 4.0: an outlook, https://doi.org/10.1155/2016/3159805, Int. J. Distrib. Sens. Netw., 12(1), 1-10 (2016)

Wilson, H.J., y Daugherty, P.R., Collaborative intelligence: humans and Al are joining forces, Harvard Business Review, ISSN 0017-8012, 96(4), 114-123 (2018) 